\title{
On the Traditional Letters and E-mail Relationship
}

\author{
Zhiqiang Yu \\ College of literature and Journalism, Shandong University of Technology \\ Zibo 255049, Shandong, China \\ E-mail: yuzhq@sdut.edu.cn
}

\begin{abstract}
The emergence of the Internet has enabled us to communicate more easily and conveniently, the emergence of E-mail makes the letter to be transferred quickly and easily, but compared with the traditional letters it also exists a certain disadvantages.
\end{abstract}

Keywords: Traditional correspondence, E-mail, Comparison, Advantage, Defects

With the development of the computer technology and network technology, more and more people's lives can not be separated from the computer than ever before .It changes our lives, to narrow our distance with the world, so we can contact with our distant friends very easily. People can even hear real-time remote friend's voice and see a friend's life images, which is our predecessors dream. However, we can still find the wisdom of our ancestors flashing in our everyday lives. Therefore, we must have innovative succession in our life. The combination of traditional letters and network technology gave birth to E-mail. E-mail correspondence in terms of traditional forms to content has undergone enormous changes.

E-mail is one kind of network tools based on the computer and communications network's information transmission service, which provides electrical signal transmission and storage of information for users to send e-mail, the document digit fax, the image, the digitized pronunciation and so on various types information. It is based on the computer as a tool, the network as the foundation, communicating information through planning to writing - - sending - - transmitting- receiving- - memorizing and other means to achieve the ultimate goal. But the traditional correspondence takes the language or the writing as a tool and the paper as the carrier, by hand delivery to achieve the ultimate purpose. We can say that E-mail is one kind of computerization correspondence shape which rises along with the computer network development, and also the modernization variant of traditional correspondence. Compared with the traditional correspondence, E-mails have the very big superiority and also certain shortcoming. This article is intended to elaborate on this issue.

\section{The advantages of E-mail correspondence compared with the traditional correspondence mainly manifested in the following areas}

\subsection{Across time and space constraints, improve work efficiency}

Traditional delivery of correspondence by natural conditions and the transportation vehicle and other detached condition's restriction, at least a few days, or a few weeks, longer if sending abroad, even the fastest Speedpost take 24 hours to mail sent to other side of the ocean. If just like Wang Shunyou's (mailman) postal route, we don't know the transmission of correspondence is how long, but he did not know how much hard work to be subject. But E-mail is carries on the transmission by the speed of light, almost in your mouse on the moment the letter served on each other's mail server, regardless of you are spatial in the ends of the earth perhaps the universe star, so long as you meet the receive requirement, you will very easy receive mail, therefore, there is no ancient " The white hair is shorter, does not use the hairpin nearly "that kind of restless anticipation feeling. E-mail almost has no concept of distance, so that people's means of communication has had a fast-growing and it easily, greatly accelerated the rhythm of life and work. In order to raise the efficiency, if we compile all friends' E-mail address of the preparation group, when send an E-mail, it can be served on all objects, so that all our friends share our good and bad in life. But the traditional correspondence Wastes the time and the strength, because we copy personally certain, and distribute one by one. 


\subsection{Forms of information various forms and of large quantities}

The traditional correspondence takes the paper as a carrier, Takes the expression form by the writing mark and the picture. But E-mail broke this kind of limit, takes the multimedia achievement as a performance method. So-called multimedia, including the writing media, the sound media (including music, pronunciation), the image media (including graph, image, animation, video), has realized the new artistic combination of different media. E-mail may insert the character pronunciation or the background music, a section of animation or video camera so the reader is possible to use visual, auditory and other sensory organs to savor the feelings of friends, moreover may they according to need to freely choose the media. The E-mail author's and receiver even may carry on the interactive communication through the media. The hypertext way's utilization, causes us to carry on the explanation, instruction, answer the question, and provide author's cultural background and the writing motivation become very easy. The hypertext is an open structure, is one kind of interconnection data, and it is mainly composed of the node and the chain. The information is stored in the node, the context relations are expressed by the chain. So long as the reader clicks on a color phrase or graphics by the mouse, the browser will jump to another location or another super-text. The hypertext is similar to human's netted memory structure. It uses the misalignment network structure to organize massive information, without having the permanent order. This information system is like the overlap of a total informative, multi-dimensional, several heavy space and time. Network Storage, Annex paste and other ways provide the storage space for us, facilitate information transmission, the memory and the extraction, but the traditional correspondence text structure is closed , and is unable to extend, its information content is also unable to compare with the E-mail.

\subsection{Saving a great deal of resources and saving a lot of money}

The traditional correspondence's transmission needs to establish a huge organization, for example, it must establish the every large or small post offices, put in the massive manpower and resources, like transportation vehicle, administrative personnel, mailman and so on, and also the envelope, the paper, the postage stamp and so on, the fund spent is a huge digit, but we send E-mail at an expense less than 1 cent money one time. The traditional letter's postage is 0.6 Yuan one time in this city, the outside areas 0.8 Yuan, several Yuan toward overseas, if the overload postage must be double. But Surfer expense is 45 Yuan one month, if the entire day accesses the net, then only uses 1.5 Yuan every day. Along with the technical progress, his expense must be reduced. However sending an E-mail, probably needs dozens of seconds, spends 1 cent money, moreover has no much length limitation.

\subsection{Accurate, confidential}

Because the transmission process of the traditional correspondence $\mathrm{s}$ is complex, the middle link are too many, there are problems sometimes. For example, lost, forgot or others private opens and so on, and it will bring the very big loss for the letter author and the receiver. While E-mail has a special transmission path, since the depositing mailbox also needs a password to open, which avoids the traditional correspondence's some possible shortcomings. E-mail may also hide the information transmission's specific address, the address is only a contact mark. It has demonstrated the specific positioning bit world, but not the primitive world geographic coordinate, so it is difficult to determine the specific position of the author. As long as you have one computer terminal to connect the Internet network, no matter where you are, as long as you enter the E-mail address correctly, it will be very loyal to pass the information in a second to the other side, and meanwhile, you can also accept any E-mail from any place. As long as your friends may contact through the E-mail address with you, but they are actually unable to know your physical location, so that you avoid any person to disturb, have a free space for leisure without any disturbance.

\subsection{To bring enormous business opportunities to businessmen}

First of all, as a network operator provides network services, through a variety of ways to attract customers, to attract consumer's spending. Commodities operators also seize this channel to sell their own goods. So when we open the website and land own mailbox, we will find all kinds of ads filled in our vision, of course, we also browse the products we care about, or even directly from the online do shopping. It is more than the traditional correspondence on the envelope attractive, and wider range of transmission. The E-mail service's tender creation's advertisement benefit can not be compared with the traditional correspondence. Therefore, E-mail facilitates the commercial activities greatly.

\section{Defects of E-mail correspondence compared with the traditional correspondence mainly manifested in the following areas}

\subsection{Network language's snack-rization makes people's feelings too explicit}

The traditional correspondence is a kind of essential tool for human being in the field of study, work, life and in human communication in the real life. It requests lucidity, accuracy for good effects, in order to achieve the good effect. An author wrote to his her relatives and friends, not only may transmit his/her own thoughts and feelings, give the recipient of letter a warm felling of "on seeing this letter as if talking to you face to face", but also both can make sides have a kind of anticipation feeling in the correspondence contact. Meanwhile as both sides can only use language or images to exchange feelings, the hazy and ambiguity of language makes the author keep the blank in the language aspect in order 
to let the reader carry on the imagination and the association. Therefore the authors need to work at dition and structure with special care, in order to move and touch by its sentiment, and matter, and hope to achieve the effect of" Grieved over the years, flowers make us shed tears; Hating to part, hearing birds breaks our heart." And readers will also be anxious about author's urgency, and be sensitive to the feelings of author. Realize "the most moving is emotional, language, sound and moral."

E-mail receives the quick rhythm of the network culture and the multimedia method's influences, its language style presents the simple and brief characteristic, the emotion too straight dew. E-mail writing has a fixed pattern, makes people always the same feeling. The signifying writing's application of sign language promotes, raised the written speed, saves time and space, and is also cost-saving, People use symbols and abbreviations briefly to replace some complex things such as feelings and images to accelerate the speed of information transmission. For example: -) expresses the ordinary smiling face, @ >>-->-- expresses bunch of attractive roses. LOL is "Laugh out loud'. Is laughing", VG is "I am Very Good". Application of symbolic language is precisely its emotional explicit disadvantages. The words in the hypertext are frequently, presented discolorative bodies. They have the link function, and are the hypertext open produces the spot. Clicks on these characters, you can enter into a new document which is related. It is precisely because the node of discolorative characters enables E-mail with a hypertext open mesh structure, and to obtain an unlimited connotation theme. Infinite extends simultaneously also enables the reader to drift away from the theme the text, inquires into asks of the source, so dilute the people's feelings and waste readers' time .The multimedia applications cause the sense of hearing and the vision at the information dissemination synchronize disturbance, thus sometimes Audio-visual communication channels is not as good as only leading to a sensory as effective channels of communication. Therefore, multi-media works of the more vivid, the image of concrete, then the reader's the space of imagination world be smaller. In this sense, the traditional correspondence through the imagery and metaphor and other means to fully mobilize the main recipients of the creative imagination is better than multi-media works.

\subsection{Network virus bring us harm}

Compared with the traditional correspondence the biggest harm for e-mail is network virus. Network virus is a kind of faster, more harmful computer viruses. Such as " kills the virus beautifully ", it has demonstrated the new characteristic of attacking method: Taking advantage of people's most common network services - e-mail and word processing software WORD attack. " kills the virus beautifully "'s dissemination way is no longer through the floppy disk copy, but through the network transmission of e-mail message conveyed in the annex to fast networks. When the user opens the e-mail and activate it, the virus in a user's computer address book of the communications collects the first 50 addresses, then sends out e-mail. It is trouble. Although it seems only the practice of a virus hoax, but in fact with some simple transformation, it is entirely possible to collect to the user computer's secret information, and sends it through e-mail. The virus itself has already been the headachy question. However, along with the pioneering Internet development, the network virus appeared, it is the virus which disseminates in the network and brings the disastrous consequence for the network. In short, network viruses like a hateful guy, after he puts on "e-mail"'s the coat, makes us in using so cute "e-mail" have some misgivings. These worries are not in the traditional correspondence.

\subsection{It is difficult to appear as tradition letter form's literature competitive products}

The network literature has brought changes for the new time's literature from the content to the form, but also has brought the unprecedented impact on the correspondence, then the literature commentary's standard cannot be irrevocable. Therefore, Wang Meng has boldly predicted that it can also appear literary master in the future, but in his imagination, present's master will perhaps not be like Mr. Lu Xun, but it will be sits peacefully there, and whisper to the reader gently through the network. Therefore we cannot use the original standard to the correspondence to judge whether is the high-quality goods, from Wang Meng's enlightenment we can imagine that E-mail will also with appear the high-quality goods in the future, but will not be our tradition form, perhaps as Wang Meng said. But, it will give us a little bit sorry, will let the human be reluctant to part with the traditional culture, and will miss traditional culture.

In short, E-mail is a new combination of technology and literature and the emergence of a new style of application. Like the tradition of practical, it has both advantages and shortcomings, which is a very normal phenomenon, so we should treat it correctly, carefully protected. It can not be immersed in the memories of traditional, to left obliterate its tremendous role. I firmly believe that with the continuous development of network technology, E-mail will also usher in a new spring to play a greater role.

\section{References}

Bai, Juyi (Tang Dynasty). Yu zhu yuan jiu shu in Bai, Juyi, Baijuyiquanji. Tianjin: Tianjin Classics Publishing House, pp. 770.

Du, Fu (Tang Dynasty). Chun wang in Du, fu, Dufuquanji. Shanghai: Shanghai Classics Publishing House, pp. 269. 